\title{
Alternative Reagents for Chemical Noise Reduction in Liquid Chromatography-Mass Spectrometry Using Selective Ion-Molecule Reactions
}

\author{
Xinghua Guo, ${ }^{\mathrm{a}}$ Andries P. Bruins, ${ }^{\mathrm{b}}$ Thomas R. Covey, ${ }^{\mathrm{c}}$ \\ Martin Trötzmüller, ${ }^{a}$ and Ernst Lankmayr ${ }^{a}$ \\ ${ }^{a}$ Institute of Analytical Chemistry and Radiochemistry, Graz University of Technology, Graz, Austria \\ ${ }^{\mathrm{b}}$ Mass Spectrometry Core Facility, University of Groningen, Groningen, The Netherlands \\ c Applied Biosystems/MDS Sciex, Concord, Ontario, Canada
}

Reduction of ionic chemical background noise based on selective gas-phase reactions with chosen neutral reagents has been proven to be a very promising approach in liquid chromatography-mass spectrometry (LC-MS). In this study further investigations on alternative reagents including the disulfides (dimethyl disulfide, diethyl disulfide, methyl propyl disulfide), dimethyl trisulfide, ethylene oxide, and butadiene monoxide, for example, have been carried out. Tandem mass spectrometric studies of ion/molecule reactions indicate that-besides dimethyl disulfide - ethylene oxide and butadiene monoxide also exhibit very efficient reactions with background ions. Furthermore, it is confirmed that the reactions are very selective according to the test with some analyte ions. In contrast to its rapid reactions with background ions, ethylene oxide does not react, or reacts much less, with these analytes. Therefore, it can be used as an alternative reagent for noise reduction. Although reactions of the other tested neutral reagents with background ions are evaluated, they are generally not suitable as reagents for this purpose because of lack of reactivity or dramatic ion losses during reactions. (J Am Soc Mass Spectrom 2009, 20, 105-111) (c 2009 Published by Elsevier Inc. on behalf of American Society for Mass Spectrometry

$\mathrm{T}$ The interference and reduction of chemical background noise have been active topics of investigations since the inception of atmospheric pressure ionization mass spectrometry (API MS) [1, 2]. Chemical background ions [3] are inherent by-products of electrospray ionization (ESI) [1] and atmospheric pressure chemical ionization (APCI) [2], partially resulting from the soft, efficient, and generic nature of ionization. Any traces of ionized contaminants or "stable" cluster ions (because the ions survive under mild declustering conditions) can be detected as sources of chemical background noise. Nowadays the interference is still commonly observed in advanced liquid chromatography-mass spectrometry (LCMS) systems even when an improved interface for declustering and the proper high-purity LC solvents/additives are applied [4]. Severe effects on limits of detection and quantitation in quantitative studies as well as in qualitative identifications [5] of unknowns are still frequently encountered.

Most hardware approaches of noise reduction [6] focused on improving ionization selectivity, declustering (desolvation) conditions, ion transmission [7], pre-

Address reprint requests to Dr. Xinghua Guo, Graz University of Technology, Institute of Analytical Chemistry and Radiochemistry, Technikerstrasse 4, 8010 Graz, Steiermark, Austria. E-mail: X.guo@tugraz.at venting contamination from ion sources and tubing, and so forth. The comprehensive tandem mass spectrometry (MS/MS) scan modes, such as selected ion monitoring, multiple reaction monitoring, neutral loss scan, product ion scan, and precursor ion scan, can further improve the MS detection specificity. However, these MS/MS techniques are generally not suitable for trace analysis of unknowns, where analyte signals are generally as low as the chemical background ions in a full-scan mode.

Enhancement of the signal-to-noise ratio $(\mathrm{S} / \mathrm{N})$ can also be achieved by reducing the level of background noise. The recent application of high-field asymmetry ion mobility spectrometry (FAIMS) [8] as an interfacing technique [9] in LC-MS is also aimed at the reduction or separation of ionic chemical noise to improve sensitivity. In addition to these hardware improvements, various off-line processing software programs have been developed and implemented into some commercial systems, such as the component detection algorithm (CODA) [10], the matched filtration with experimental noise determination [11], the sequential paired covariance [12], and other noise filtration methods [13]. Other software-assisted approaches such as dynamic background subtraction [14], active chemical background noise reduction [15], and windowed mass selection [16]
(C) 2009 Published by Elsevier Inc. on behalf of American Society for Mass Spectrometry. 1044-0305/09/\$32.00

doi:10.1016/j.jasms.2008.09.021
Published online September 30, 2008 Received July 3, 2008 Revised September 1, 2008 Accepted September 7, 2008 
have also been applied as on-line noise reduction techniques. However, the above-mentioned techniques have not really solved the problem of interference from chemical background noise [5]. The best solution is probably the selective removal or reduction of chemical noise ions before they reach the MS detector, rather than post-acquisition data processing.

In our previous study we have developed a novel noise-reduction technique $[17,18]$ based on selective reactions of the neutral reagent dimethyl disulfide (DMDS) with background ions in LC-MS, where apparently the significant structure characteristics of ionic noise [4], compared with that of conventional protonated species, have played a crucial role resulting in different reactivities. Both the cluster-type background ions and fragment intermediates of contaminants react very selectively with DMDS to form products with a different $\mathrm{m} / \mathrm{z}$ value. It has been demonstrated that reduction of the base-peak chromatographic baseline intensity by up to 40 -fold and improvement of $\mathrm{S} / \mathrm{N}$ by 5- to 10-fold have been achieved on commercial LCtriple quadrupole mass spectrometer. This is mainly attributed to the chemical resolution resulting from the selective reactions between background ions and the reagent gas. In a separate study in inductively coupled plasma (ICP)-MS it has also been concluded [19] that the use of ion/molecule chemistry is more efficient than that of collisional dissociation/cooling. However, the choice of a suitable reagent for noise reduction is a determining factor. This arises from the unpredictable chemistry involved, although structures of some background ions [4] and their reaction pathways with DMDS [20] have been studied. In this report we present a preliminary investigation on alternative reagents for noise reduction. The tested compounds include the structure analogues of DMDS, i.e., two disulfides and one trisulfide, and ethylene oxide and butadiene monoxide.

\section{Experimental}

MS and LC-MS experiments were mainly carried out on custom-modified API365 and commercial API2000 triple quadrupole mass spectrometers (MDS Sciex, Concord, ON, Canada) coupled with an LC system. For comparison, some experiments were also performed on a Sciex API3000 LC-MS. The collision gas inlet of the mass spectrometers was modified to introduce a volatile chemical reagent into the radio-frequency (RF)only quadrupole (Q2) collision cell. This modification allows freeze, pump, and thaw cycles needed to degas the liquid chemical reagents and a quick switch between the chemical reagent and the normal collision gas nitrogen [18].

Various LC eluents as described in the following text with different organic compositions, additives, elution conditions (gradient and isobaric), and MS ionization and acquisition modes were investigated with regard to ionic chemical background noise (LC column: Vydac
$\mathrm{C}_{18}, 200 \times 2.0 \mathrm{~mm}$ i.d., $5 \mu \mathrm{m}$ ). The LC flow rate was 100 $\mu \mathrm{L} / \mathrm{min}$. Positive ESI ionization was used.

Details of similar experiments have been published previously [18]. Briefly, to study the gas-phase reactions, ions of the background interference or the protonated analytes were first mass-selected by Q1 and then allowed to undergo reactive collisions with the reagent present in the second quadrupole Q2 (RF-only). The amount of the reagent introduced into the cell was adjusted by means of a needle valve and was monitored by the readout of a Bayard Alpert gauge mounted in the vacuum chamber of the mass spectrometer, which is close to the turbo molecular pump. Under normal Q1-scan operating conditions it is about $0.6 \times 10^{-5}$ torr. When the neutral reagent was introduced the pressure readout increased to $1.3 \times 10^{-5}$ torr. Here the increment $\left(0.7 \times 10^{-5}\right.$ torr in this example) is called the "partial pressure" of the reagent. The experimental setup did not allow a direct pressure measurement inside the collision cell, which is estimated to be a few millitorr. The resulting ion/molecule reaction mixture was monitored by scanning the mass-resolving quadrupole Q3.

To study the reduction of chemical interferences through full-scan mass spectra, the mass spectrometer was operated in the zero neutral loss (ZNL) scan mode and with the chemical reagent present in the Q2 collision cell, whereas the LC was run in the normal mode and conditions. The ZNL scan is a special case of the well-known constant neutral loss mode in MS/MS with the neutral loss set to zero. The optimized collision energy of $3-5 \mathrm{eV}$ was used, which is a compromise between ion transmission and reaction efficiencies. A lower energy could probably allow more efficient reactions but not all products would be transmitted to the detector. In addition, the dwell time was set to $5-10 \mathrm{~ms}$, whereas no significant effect is observed above $5 \mathrm{~ms}$. For comparison, the conventional collision gas $\mathrm{N}_{2}$ was also used in some experiments to acquire normal Q1, Q3, and ZNL scan data for both infusion and LC-MS studies.

The chemical reagents dimethyl disulfide $\left(\mathrm{CH}_{3} \mathrm{~S}-\mathrm{SCH}_{3}\right.$, DMDS, 99\%), disulfides (diethyl disulfide, methyl propyl disulfide), dimethyl trisulfide, and butadiene monoxide were purchased from Acros Organics (Geel, Belgium). Ethylene oxide $(99.8 \%)$ was from Sigma-Aldrich. The tested analyte compounds and the LC grade solvents and additives were commercially available and used as received. For infusion, a stock solution of analytes at a final concentration about 5-50 $\mu \mathrm{M}$ was used.

\section{Results and Discussion}

\section{Reduction of Chemical Background Noise Using Selective Reactions with DMDS}

In our recent study $[17,18]$ DMDS has been discovered to react very selectively with the vast majority of background ions and applied as the first neutral reagent to reduce chemical noise in API-LC-MS while it is 
combined with the mass filtering functions of quadrupole analyzers in MS/MS mode. Under the conventional ZNL or Q-3 full-scan MS condition, some clustertype background noise may also undergo dissociation reaction during collisions if the collision energy is sufficiently high [21]. However, in our approach [18], the collision energy has been kept sufficiently low to prevent dissociation of any analytes. It has been proven that noise reduction with DMDS is predominantly the result of selective reactions with background ions. A typical experimental setup and comparison of the resulting LC-MS total ion current (TIC) chromatograms and mass spectra are given in Figure 1. This control assay was done by switching the reagent DMDS and the conventional collision gas $\mathrm{N}_{2}$ at the same partial pressure of $0.7 \times 10^{-5}$ torr (that is about the collision gas pressure needed for a normal MS/MS operation) during one LC-MS run, as shown in Figure 1a. The LC mobile phase was kept isocratic [0.1\% formic acid in 1:1 acetonitrile (ACN)/water, vol/vol] to obtain constant chemical background noise for comparison. When DMDS was used for noise reduction, the level of the TIC baseline drops to about $1 / 9$ th compared to that with $\mathrm{N}_{2}$ (Figure 1a), which indicates that the chemical reactions of DMDS with background ions are the main reason for the efficient noise reduction rather than ion losses by collisions. Furthermore, the significant decrease of the baseline for the period without any gas in the cell at 10-20 min (again compared to that with $\mathrm{N}_{2}$ at 30-40 min) demonstrates that, at this pressure range, the collision gas (and the reagent) in the collision cell assist ion transmission by means of collisional focusing [22]. It is estimated that about $90 \%$ of the original chemical background ions were removed with the use of DMDS. The corresponding normalized mass spectra of these three experimental periods with DMDS, without gas, and with $\mathrm{N}_{2}$ are given in Figure $1 b, c$, and $d$, respectively. The dramatic reduction of the intensities of almost all background ions (from Figure $1 \mathrm{~d}$ to $1 \mathrm{~b}$ ) is observed accordingly. In our previous study it has been confirmed that the reactions are mainly rapid clustering of DMDS with background ions, which shifts the massto-charge ratio $(\mathrm{m} / \mathrm{z})$ of these ions and is the basis for the noise-reduction technique $[17,18]$. Note that the reduction is not proportional for all ions, but is differently distributed among them because of the various reactivities of the background ions with DMDS.

Besides dimethyl disulfide other compounds, such as disulfides (diethyl disulfide and methyl propyl disulfide) and a trisulfide (dimethyl trisulfide), were also investigated briefly. Compared to dominating clustering reactions of DMDS with background ions to form
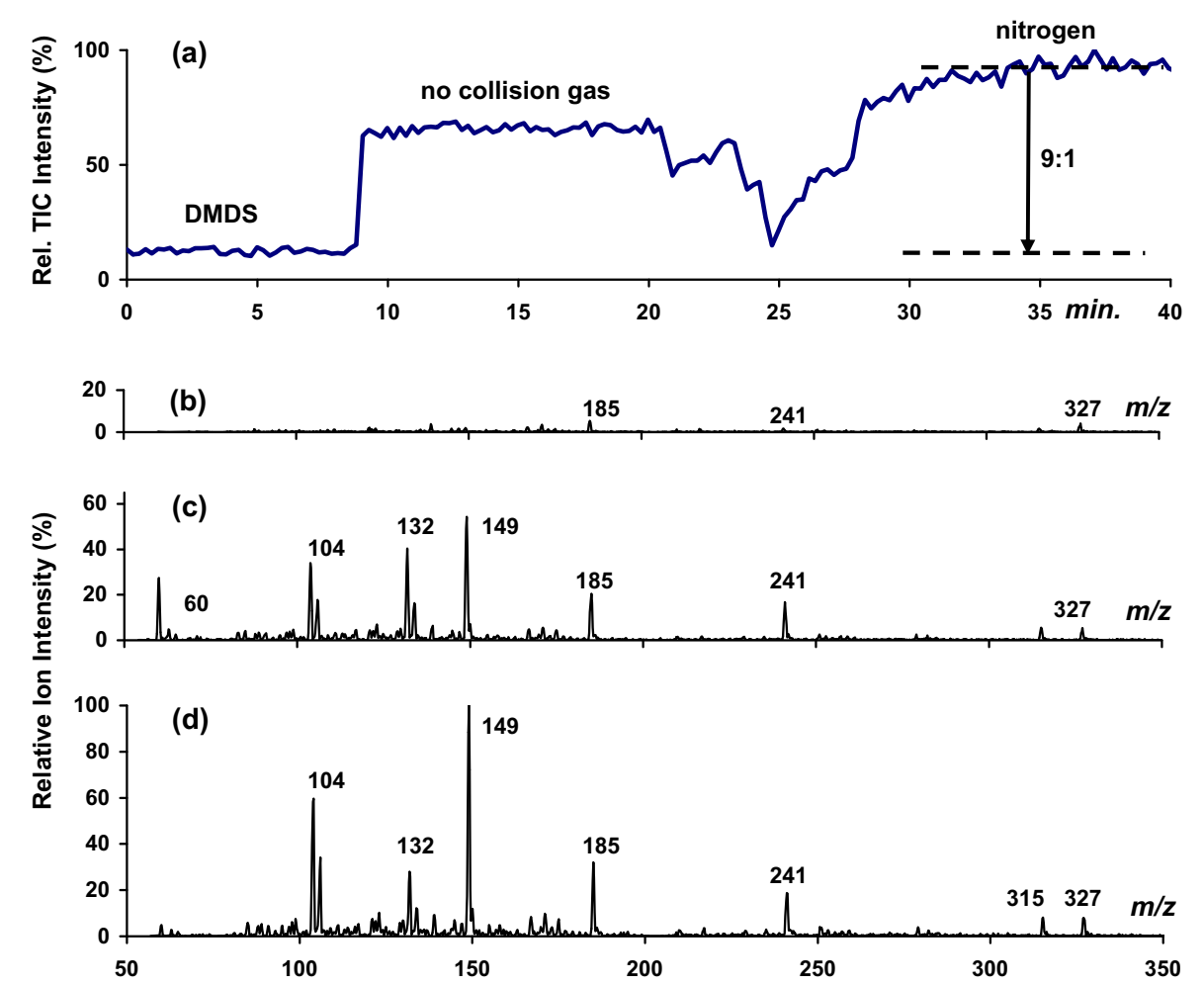

Figure 1. (a) Changes of the ZNL TIC baseline noise level when the collision gas is switched from reactive reagent DMDS $(0-8.5 \mathrm{~min})$ to $\mathrm{N}_{2}(30-40 \mathrm{~min})$ at the partial pressure of $0.7 \times 10^{-5}$ torr. For comparison, the baseline for the period, when no collision gas (therefore, only the vacuum background pressure of $0.6 \times 10^{-5}$ torr) was introduced into the collision cell (10-20 $\mathrm{min}$ ), is also included. The resulting normalized background mass spectra are shown as follows: (b) with DMDS, (c) without any collision gas, and (d) with $\mathrm{N}_{2}$, respectively. Chemical background ions were generated from the mobile phase $0.1 \% \mathrm{HCOOH}$ in $\mathrm{ACN} / \mathrm{H}_{2} \mathrm{O}(1: 1 \mathrm{vol} / \mathrm{vol})$. 


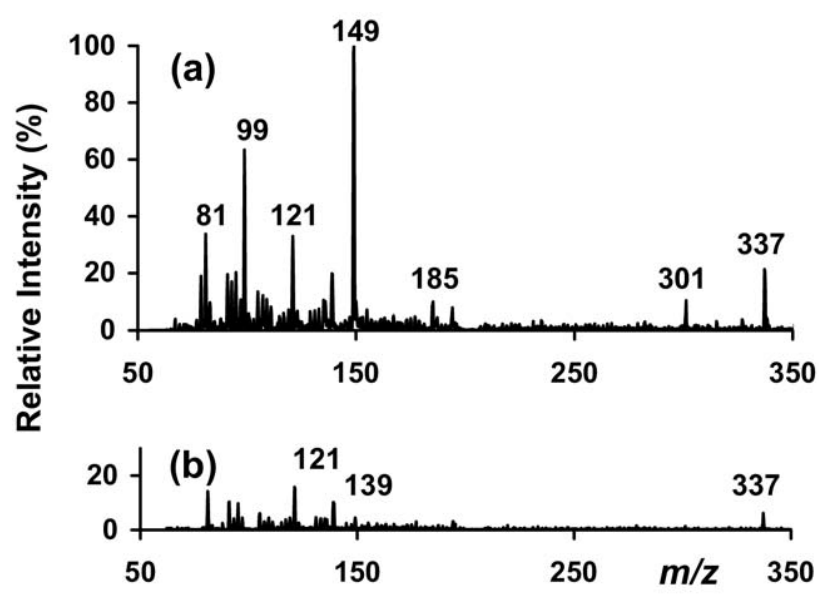

Figure 2. Comparison of the change of the normalized background mass spectra (ZNL scan mode) when ethylene oxide (a) and $\mathrm{N}_{2}(\mathrm{~b})$ were used in the collision cell. Chemical background ions were generated from the mobile phase of $0.1 \% \mathrm{AcOH}$ in $\mathrm{MeOH} / \mathrm{H}_{2} \mathrm{O}$ (1:1 vol/vol).

[background $\left.+(\mathrm{DMDS})_{n}\right]^{+}$, these di- and trisulfides induce charge abstraction, producing ionized di- and trisulfides followed by reactions with one more neutral di- or trisulfide, where the cleavage of $S-S$ bonds is apparently one of the favorite pathways. Only a few background ions react to form clusters [background + sulfide $]^{+}$. Most of the di- and trisulfides are eliminated from a further study because of low reactivity, lower volatility, or significant loss of analyte ions during low-energy collisions.

\section{Efficient Reactions of Ethylene Oxide (EO) with Chemical Background Ions}

Similarly to DMDS, ethylene oxide $\left(\mathrm{c}-\mathrm{CH}_{2} \mathrm{CH}_{2} \mathrm{O}\right)$ demonstrates very efficient reactions toward the vast majority of the background ions. The level of the TIC noise baseline can be lowered to about $1 / 5$ th to $1 / 10$ th, depending on the EO partial pressure applied. A comparison of the changes of the typical normalized mass spectra, when EO and $\mathrm{N}_{2}$ (partial pressure $0.7 \times 10^{-5}$ torr) were used in the collision cell, is given in Figure $2 \mathrm{a}$ and $\mathrm{b}$, when $0.1 \%$ acetic acid $(\mathrm{AcOH})$ in $\mathrm{MeOH} /$ water $(1: 1, \mathrm{vol} / \mathrm{vol})$ was used as the mobile phase. Both the disappearance of the background ions and the reduction of their abundances are a result of reactions with $\mathrm{EO}$.

Compared to DMDS, the reactions of EO with both cluster-type ions and contaminants are much faster and undergo multiple steps and pathways, which are nevertheless still dominated or initiated by clustering with neutral EO molecules. The mass spectra of the reactions of the ion $\left[\mathrm{H}_{3} \mathrm{PO}_{4}+\mathrm{H}\right]^{+}(\mathrm{m} / z$ 99) and its acetic acid clusters $\left[\mathrm{H}_{3} \mathrm{PO}_{4}+\mathrm{AcOH}+\mathrm{H}\right]^{+}(m / z 159)$ and $\left[\mathrm{H}_{3} \mathrm{PO}_{4}+\right.$ $\left.(\mathrm{AcOH})_{2}\right]+\mathrm{H}^{+}(m / z 219)$ are shown in Figure 3. Up to five stepwise additions of $\mathrm{EO}$ to $\left[\mathrm{H}_{3} \mathrm{PO}_{4}+\mathrm{H}\right]^{+}$

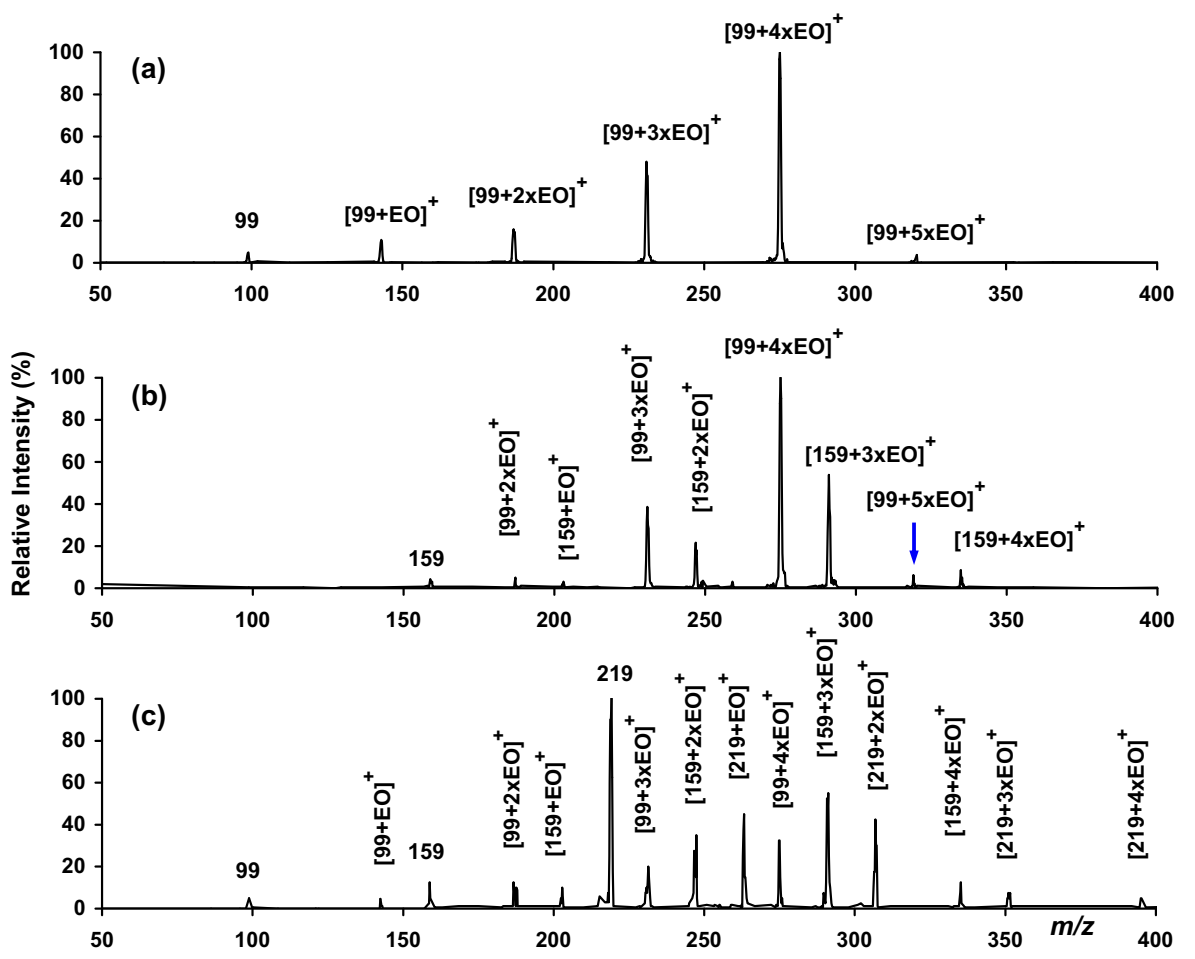

Figure 3. Mass spectra of the reactions of the chemical noise ions $\left[\mathrm{H}_{3} \mathrm{PO}_{4}+\mathrm{H}\right]^{+}$at $\mathrm{m} / \mathrm{z} 99$ (a), its clusters $\left[\mathrm{H}_{3} \mathrm{PO}_{4}+\mathrm{AcOH}+\mathrm{H}\right]^{+}$at $m / z 159(\mathbf{b})$ and $\left[\mathrm{H}_{3} \mathrm{PO}_{4}+(\mathrm{AcOH})_{2}+\mathrm{H}\right]^{+}$at $m / z 219$ (c) with the reactive collision gas $\mathrm{EO}$ at the partial pressure of $0.7 \times 10^{-5}$ torr. The background pressure before introducing $\mathrm{EO}$ was $0.6 \times 10^{-5}$ torr. Chemical background ions were generated from the mobile phase of $0.1 \% \mathrm{AcOH}$ in $\mathrm{MeOH} / \mathrm{H}_{2} \mathrm{O}(1: 1 \mathrm{vol} / \mathrm{vol})$. 
forming $\left[\mathrm{H}_{3} \mathrm{PO}_{4}+(\mathrm{EO})_{n}+\mathrm{H}\right]^{+}(n=1-5)$ were observed as indicated in Figure 3a. Among the products, the ion $\left[\mathrm{H}_{3} \mathrm{PO}_{4}+(\mathrm{EO})_{4}+\mathrm{H}\right]^{+}(\mathrm{m} / \mathrm{z} 275)$ is apparently the predominant end product. It suggests that it may have a relatively stable structure compared to that of $\left[\mathrm{H}_{3} \mathrm{PO}_{4}+(\mathrm{AcOH})_{m}+(\mathrm{EO})_{n}+\mathrm{H}\right]^{+}$ ions as shown in Figure 3. Addition of up to four EO molecules has been observed for the other two ions $\left[\mathrm{H}_{3} \mathrm{PO}_{4}+(\mathrm{AcOH})_{n}+\mathrm{H}\right]^{+}(\mathrm{m} / \mathrm{z} 159$ and 219) followed by extensive dissociation of the products (i.e., ligand exchange by loss of neutral acetic acid molecules). The latter process is quite different from that of DMDS [18, 20], where the cluster-type noise ions undergo clustering reactions with DMDS and water. Furthermore, some other ions exchange charges with DMDS and, finally, form the common product ion [DMDS $\left.+\mathrm{SCH}_{3}\right]^{+}$[23]. Other cluster noise ions containing water molecules demonstrate a similar reactivity with $\mathrm{EO}$. The typical mass spectra of reaction products of $\left[\mathrm{H}_{3} \mathrm{PO}_{4}+\mathrm{H}_{2} \mathrm{O}+\mathrm{H}\right]^{+}(\mathrm{m} / z$ 117) and $\left[\mathrm{H}_{3} \mathrm{PO}_{4}+\mathrm{H}_{2} \mathrm{O}+\mathrm{AcOH}+\mathrm{H}^{+}{ }^{+}(m / z\right.$ 177) with EO are given in Figure 4, where $\left[\mathrm{H}_{3} \mathrm{PO}_{4}+(\mathrm{EO})_{4}+\mathrm{H}\right]^{+}(\mathrm{m} / \mathrm{z}$ 275 ) is once again the most abundant of the product ions. In this study it is not clear whether an EO molecule would open its ring structure to initialize polymerization-type reactions. Further investigation on kinetics and mechanisms can be very interesting for understanding these reactions.

Most of the background ions resulting from contaminants [4] react efficiently with EO with much less dissociation of the product ions. The ions from phthalates, for instance, also exhibit the reactivity of catching up to two EO neutral molecules, which is nevertheless dominated by the addition of a single EO molecule. This includes protonated phthalic anhydride at $\mathrm{m} / \mathrm{z} 149$, protonated phthalate/water clusters at $m / z 185$ and 241, and the sodiated phthalate $m / z 301\left(=[278+\mathrm{Na}]^{+}\right)$and $413\left(=\left[390+\mathrm{Na}^{+}\right)\right.$. Their relative reactivities-that is, the percentage of the product ions relative to the total precursor ion abundance-are estimated (with an uncertainty of about $\pm 20 \%)$ to be $86(\mathrm{~m} / \mathrm{z} 149), 80(\mathrm{~m} / \mathrm{z} 185)$, $58(\mathrm{~m} / \mathrm{z} 241), 61(\mathrm{~m} / \mathrm{z} 301)$, and $61 \%(\mathrm{~m} / \mathrm{z} 413)$, respectively. The ions at $\mathrm{m} / \mathrm{z} 149$ constitute a well-known characteristic for contamination from phthalate plasticizers [4]. They could also be viewed as a substituted acylium ion $[\mathrm{Ph}-\mathrm{C}=\mathrm{O}]^{+}$that undergoes the gas-phase Meerwein reaction [24, 25], as reported by Eberlin and Cooks. The reaction results in a stable product through three- to five-membered ring expansion and can explain the reason for the dominance of a single addition of EO. This mechanism may also be involved in reactions of EO with other contaminant types of background ions reported here. However, it is still not clear for reactions of those cluster-type background ions discussed earlier, where multiple additions of EO are always observed. In conclusion, all background ions demonstrate sufficient reactivities for the purpose of the noise reduction studied here.

In addition, the EO's structure analogue, butadiene monoxide (BO), also reacts efficiently with most background ions. For instance, up to four $\mathrm{BO}$ molecules with $\left[\mathrm{H}_{3} \mathrm{PO}_{4}+\mathrm{H}\right]^{+}(\mathrm{m} / \mathrm{z} 99)$ and $\left[\mathrm{H}_{3} \mathrm{PO}_{4}+\mathrm{AcOH}+\mathrm{H}\right]^{+}(\mathrm{m} / \mathrm{z}$ 159) from $\mathrm{MeOH} /$ water and three with $\left[(\mathrm{ACN})_{2}+\mathrm{Na}\right]^{+}$ $(m / z$ 105) from ACN/water were observed (spectra not shown), which is very similar to the reactions of EO.

\section{Limited Reactions of Ethylene Oxide with the Selected Analyte Ions}

Our recently developed chemical noise-reduction technique is based on selective reactions of a neutral reagent

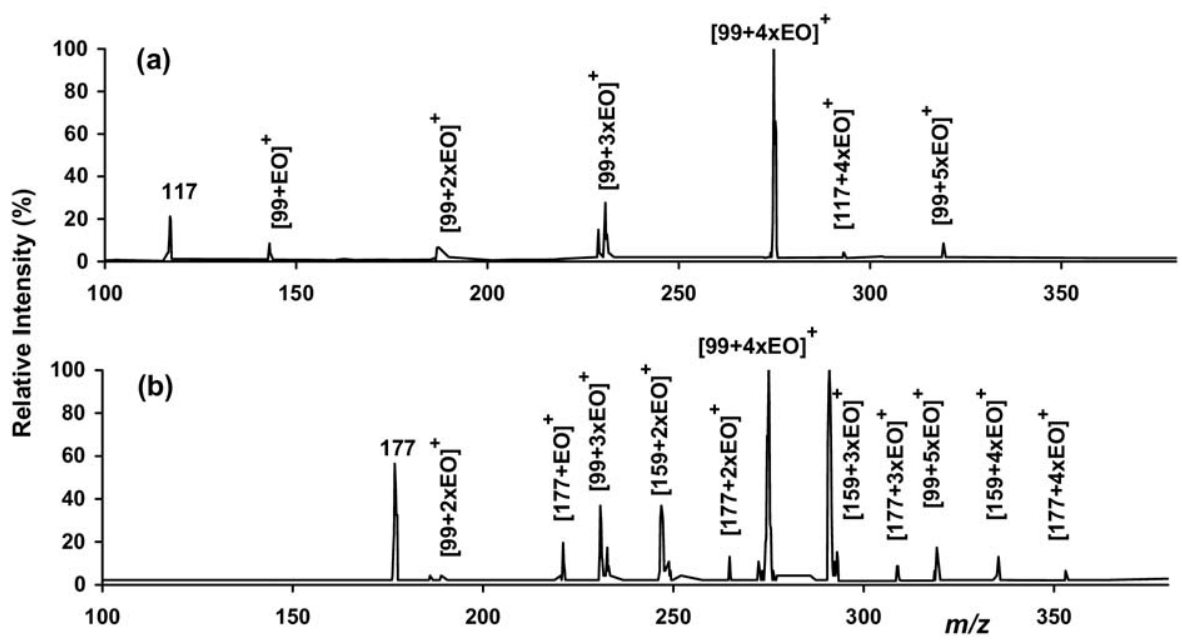

Figure 4. Mass spectra of the reactions of the cluster noise ions $\left.\left[\mathrm{H}_{3} \mathrm{PO}_{4}+\mathrm{H}_{2} \mathrm{O}+\mathrm{H}\right)\right]^{+}$at $\mathrm{m} / z 117$ (a) and $\left[\mathrm{H}_{3} \mathrm{PO}_{4}+\mathrm{H}_{2} \mathrm{O}+\mathrm{AcOH}+\mathrm{H}\right]^{+}$at $\mathrm{m} / z 177(\mathbf{b})$ with the reactive collision gas $\mathrm{EO}$ at the partial pressure of $0.7 \times 10^{-5}$ torr. The background pressure before introducing $\mathrm{EO}$ was $0.6 \times 10^{-5}$ torr. Chemical background ions were generated from the mobile phase of $0.1 \% \mathrm{AcOH}$ in $\mathrm{MeOH} / \mathrm{H}_{2} \mathrm{O}(1: 1$ $\mathrm{vol} / \mathrm{vol})$. 
with background ions [18]. Candidate reagents always need to be tested for possible reactions with analytes, which is also to demonstrate how generic the application could be, with regard to ranges of analytes. In contrast to the extensive and efficient reactions of ethylene oxide with the majority of background ions as shown in the previous section, there is generally a low reactivity of EO toward protonated analytes. For instance, under our experimental conditions, only $15 \%$ of protonated ofloxacin reacts with $\mathrm{EO}$ to form products at $\left[\mathrm{M}+(\mathrm{EO})_{n}+\mathrm{H}\right]^{+}(n=1$ and 2$)$ together with some minor dissociation products $\left[\mathrm{M}-\mathrm{H}_{2} \mathrm{O}+\mathrm{H}\right]^{+}$and $[\mathrm{M}-$ $\left.\mathrm{CO}_{2}+\mathrm{H}\right]^{+}$attributed to collisions. As a brief summary for these protonated analytes, the reactivities of several other tested compounds lorazepam, diazepam, flunitrazepam, nordiazepam, decarboxyl ofloxacin, norfloxacin, and clonazepam are estimated to range from 2 to $15 \%$. That for prazepam is about $27 \%$ but with significant dissociations. The result is very similar to that of our previous study on DMDS [18]. Although this is not a comprehensive survey, it already includes the compounds with different functional groups, proton affinities (basic to acidic compounds), and molecular masses. As a general conclusion, protonated analyte ions $[\mathrm{M}+\mathrm{H}]^{+}$tested so far result in no or very limited products, which indicates that reactions of EO with chemical background ions are rather selective. Further investigation is in progress. This indicates that $\mathrm{EO}$ can be applied as an alternative reagent for chemical noise reduction.

It is interesting to note that, in contrast to the protonated analytes, all sodium adducts studied so far react efficiently with $\mathrm{EO}$ to form predominant products $[\mathrm{M}+\mathrm{Na}+\mathrm{EO}]^{+}$. A second addition of $\mathrm{EO}$ is also often observed. These end up with a high total reactivity ranging from 57 to $83 \%$. Since protonated analytes are more often observed in LC-MS, in fact, the difference in reactivities between $[\mathrm{M}+\mathrm{Na}]^{+}$and $[\mathrm{M}+\mathrm{H}]^{+}$may provide an effective method for selective removal of unwanted sodium adducts of analytes for studies of unknown compounds by LC-MS.

In practice, for an automatic identification or screening process with LC-MS it is important to trigger an MS/MS scan to acquire further information on analyte structures. These MS/MS experiments are performed on the base peak or the most abundant ions. However, if the intensities of the trace components are already lower than those of the major (base-peak) chemical background ions in a mass spectrum, these minor ions may not be picked up and identified for a further MS/MS experiment. The importance of very selective reactions $[17,18]$ with neutral reagents is the subsequent shift of $\mathrm{m} / \mathrm{z}$ values of background ions. However, before removing them completely, these product ions still interfere with MS or MS/MS studies at other $\mathrm{m} / \mathrm{z}$ values. A design similar to that of a dynamic reaction cell [19] used in ICP-MS to remove reactive chemical noise is likely to be one of the ideal solutions to this problem. Nevertheless, without dedicated new instrumentation and without major modifications of commer- cial triple quadrupole mass spectrometers used in this study, the ZNL [26] scan has been applied to remove the reactive background ions [17]. Briefly, after a mixture of isobaric reactive and unreactive ions pass through the first quadrupole mass analyzer (Q1), the reactive ones will react with the neutral reagent present in the collision cell to form products at a different $\mathrm{m} / \mathrm{z}$ value. The unreactive ions remain unaffected. Then the second quadrupole mass analyzer $(\mathrm{Q} 3)$ allows only the ions, which retain the original $\mathrm{m} / \mathrm{z}$, to go through it. Therefore, the reactive ions (background ions, for instance) will be filtered out because of the $\mathrm{m} / \mathrm{z}$ shifts after reactions with reagent gases.

\section{Conclusions}

As part of our continuous efforts of developing a novel noise-reduction technique in API LC-MS based on selective gas-phase ion/molecule reactions of background ions [17, 18], this study has demonstrated the existence of the alternative reagent ethylene oxide (EO) besides dimethyl disulfide (DMDS). The selective reactions are dominated by efficient consecutive clustering of (up to five) neutral EO molecules with the clustertype background ions and Meerwein three- to fivemembered ring-expansion reactions with some contaminant ions. Furthermore, EO has a low molecular mass (44 Da), which can prevent/minimize ion losses during collisions and reactions. Because EO is a gas at room temperature it is easier to handle and connect to the CAD gas line than an odorous liquid such as the previously proposed DMDS. Therefore, as a possible alternative reagent for chemical noise reduction, it offers a practical advantage. However, caution should be exercised because of the toxic nature of ethylene oxide [27]. It is expected that the discovery of alternative reagents may further improve this recently developed technique for noise reduction in LC-MS, where complementary reactivities can be beneficial when two or more reagents are used jointly in case of necessity.

In addition, it should be mentioned that, in this study, the combined use of the zero neutral loss scan and selective reactions for noise reduction on a triple quadrupole LC-MS also indicates that both mass analyzing quadrupoles (Q1 and Q3) are no longer available for conventional MS/MS scans such as multiple reaction monitoring (MRM). However, as a test of theory, the approach has shown promising progress in the development of new techniques or LC-MS instrumentations for chemical noise reduction.

\section{Acknowledgments}

This study is partially the result of a research contract between Applied Biosystems/MDS Sciex (Concord, ON, Canada) and the University of Groningen (Groningen, The Netherlands). The authors are grateful to one of the reviewers for the suggestion that the gas-phase Meerwein reactions are involved for addition of ethylene oxide to some background ions. 


\section{References}

1. Yamashita, M.; Fenn, J. B. Electrospray Ion Source. Another Variation on the Free-Jet Theme. J. Phys. Chem. 1984, 88, 4451-4459.

2. Covey, T. R.; Lee, E. D.; Bruins, A. P.; Henion, J. D. Liquid-Chromatography Mass-Spectrometry. Anal. Chem. 1986, 58, 1451.

3. Busch, K. L. Chemical Noise in Mass Spectrometry. Spectroscopy 2002, $17,32-36$.

4. Guo, X.; Bruins, A. P.; Covey, T. R. Characterization of Typical Chemical Background Interferences in Atmospheric Pressure Ionization Liquid Chromatography-Mass Spectrometry. Rapid Commun. Mass Spectrom. 2006, 20, 3145-3150.

5. Marquet, P. Is LC-MS Suitable for a Comprehensive Screening of Drugs and Poisons in Clinical Toxicology? Ther. Drug Monit. 2002, 24, 125-133.

6. Alavi, A.; Cousins, L. M.; Javahery, G.; Jolliffe, C.; Vuckovic, D. Proceedings of the 52nd ASMS Conference on Mass Spectrometry; MPH-127, Nashville, TN; 2004

7. Product brochure: API 5000 ${ }^{\mathrm{TM}}$ LC-MS/MS System, Publication No. 114BR15-02. Applied Biosystems/MDS Sciex: Concord, Canada; April 2006.

8. Purves, R. W.; Guevremont, R. Mass Spectrometric Characterization of a High-Field Asymmetric Waveform Ion Mobility Spectrometer. Rev. Sci. Instrum. 1998, 69, 4094-4105.

9. Guevremont, R.; Purves, R. W. Apparatus and Method for Desolvating and Focusing Ions for Introduction into a Mass Spectrometer. U.S. Patent 6504 149, 2003

10. Windig, W.; Phalp, J. M.; Payne, A. W. A Noise and Background Reduction Method for Component Detection in Liquid Chromatography/Mass Spectrometry. Anal. Chem. 1996, 68, 3602-3606.

11. Andreev, V. P.; Rejtar T.; Chen, H.-S.; Moskovets, E. V.; Ivanov, A. R.; Karger, B. L. A Universal Denoising and Peak Picking Algorithm for LC-MS Based on Matched Filtration in the Chromatographic Time Domain. Anal. Chem. 2003, 75, 6314-6326.

12. Muddiman, D. C.; Rockwood, A. L.; Gao, Q.; Severs, J. C.; Udseth, H. R.; Smith, R. D.; Proctor, A. Application of Sequential Paired Covariance to Capillary Electrophoresis Electrospray Ionization Time-of-Flight Mass Spectrometry: Unraveling the Signal from the Noise in the Electropherogram. Anal. Chem. 1995, 67, 4371-4375.

13. Visentini, J.; Kwong, E. C.; Carrier, A.; Zidarov, D.; Bertrand, M. J. Comparison of Softwares Used for the Detection of Analytes Present at
Low Levels in Liquid Chromatographic-Mass Spectrometric Experiments. J. Chromatogr. A 1995, 712, 31-43.

14. Le Blanc, Y., Bloomfield, N. Real-Time Dynamic Background Subtraction: Improving the Automated Ion Selection Process, Technical Note 114TN02-01. Applied Biosystems/MDS Sciex: Concord, Canada.

15. Ramsey, R. S.; Goeringer, D. E.; McLuckey, S. A. Active-Chemical Background-Noise Reduction in Capillary Electrophoresis/Ion Trap Mass-Spectrometry. Anal. Chem. 1993, 65, 3521-3524.

16. Fleming, C. M.; Kowalswi, B. R.; Apffel, A.; Hancock, W. S. Windowed Mass Selection Method: A New Data Processing Algorithm for Liquid Chromatography-Mass Spectrometry Data. J. Chromatogr. A 1999, 849, 71-85.

17. Guo, X.; Bruins, A. P.; Covey T. R. Chemical Noise Reduction for Mass Spectrometry. PCT International Patent WO 2007/092873 A2, 2007

18. Guo, X.; Bruins, A. P.; Covey, T. R. Method to Reduce Chemical Background Interference in Atmospheric Pressure Ionization Liquid Chromatography-Mass Spectrometry Using Exclusive Reactions with the Chemical Reagent Dimethyl Disulfide. Anal. Chem. 2007, 79, 40134021 .

19. Tanner, S. D.; Baranov, V. I. Bandpass Reactive Collision Cell. U.S Patent 6140 638, 2000.

20. Jarvis, M. J. Y. Koyanagi, G. K.; Zhao, X.; Bohme, D. K. Scrubbing Ions with Molecules: Kinetic Studies of Chemical Noise Reduction in Mass Spectrometry Using Ion-Molecule Reactions with Dimethyl Disulfide. Anal. Chem. 2007, 79, 4006-4012.

21. Chait, B. T.; Krutchinsky, A. N. Method and Apparatus for Improved Signal-to-Noise Ratio in Mass Spectrometry. U.S. Patent 6610 976, 2003.

22. Douglas, D. J.; French, J. B. Collisional Focusing Effects in Radio Frequency Quadrupoles. J. Am. Soc. Mass Spectrom. 1992, 3, 398-408.

23. Watkins, M. A.; WeWora, D. V.; Winger, B. E.; Kenttämaa, H. I Compound Screening for the Presence of the Primary N-Oxide Functionality via Ion-Molecule Reactions in a Mass Spectrometer. Anal. Chem. 2005, 77, 5311-5316.

24. Moraes, L. A. B.; Eberlin, M. N. The Gas-Phase Meerwein Reaction Chem. Eur. J. 2000, 6, 897-905.

25. Cooks, R. G.; Chen, H.; Eberlin, M. N.; Zheng, X.; Tao, W. A. Polar Acetalization and Transacetalization in the Gas Phase: The Eberlin Reaction. Chem. Rev. 2006, 106, 188-211.

26. Hager, J. Method for Improving Signal-to-Noise Ratios for Atmospheric Pressure Ionization Mass Spectrometry. U.S. Patent 6700 120, 2004.

27. www.osha.gov/SLTC/ethyleneoxide/. 